\title{
Characterization of a Controlled-Clearance Piston Gauge Using the Heydemann-Welch Model ${ }^{\dagger}$
}

\author{
Tokihiko KobAta*, James W. SchmidT ${ }^{* *}$ and Douglas A. Olson**
}

\begin{abstract}
A study on the characterization of a controlled-clearance piston gauge was performed. In this study, the Heydemann-Welch model was used to define the controlled-clearance piston gauge as a primary pressure gauge. The parameters needed for the model were determined from the measurements, and the characteristics of the controlled-clearance gauge were evaluated. The results obtained are as follows: (1) The best jacket pressure function to keep the clearance between the piston and cylinder constant was determined from the measurement. (2) The dimensional clearance between the piston and cylinder was estimated. (3) The uncertainty of the effective area of the piston-cylinder used for the gauge was evaluated by the function of the system and jacket pressures.
\end{abstract}

Key Words: pressure standard, controlled-clearance piston gauge, effective area, uncertainty

\section{Introduction}

Controlled-clearance deadweight piston gauges are currently used to realize high-pressure standards at national metrology institutes in the world. This paper describes a characterization of a controlled-clearance deadweight piston gauge, which was performed at the National Institute of Standards and Technology (NIST) during November 2000 to April 2001. At NIST, three controlled-clearance deadweight piston gauges, which have different operating pressure ranges, are used as primary standards to realize hydraulic high-pressure standards up to $280 \mathrm{MPa}^{1)}$. In this study, one of these primary piston gauges, which is identified as PG-67 at NIST, is selected and characterized using the method described by P. L. M. Heydemann and B. E. Welch ${ }^{2)}$. The characterization covers the pressure range from $37.5 \mathrm{MPa}$ to $288 \mathrm{MPa}$, at jacket pressures up to $76 \mathrm{MPa}$. In section 2 , the structure of a controlled-clearance gauge and the equation of the effective area of the piston-cylinder assembly as given by the Heydemann-Welch model are described. The apparatus and measuring devices used for this characterization are described in section 3 . In order to characterize the piston gauge as a primary controlled-clearance gauge, the fall-rates of the piston and the change in the system pressure generated by the jacket pressure were measured and

$\uparrow$ Presented in SICE Annual Conference 2002 in Osaka (August, 2002)

* National Metrology Institute of Japan, AIST, Tsukuba

** National Institute of Standards and Technology, Gaithersburg, MD, USA

(Received July 30, 2002)

(Revised January 9, 2003) analyzed using the Heydemann-Welch model. The fallrate measurements are shown in section 4 . The results of the change in system pressure generated by the jacket pressure are described in section 5. From the measured results, the controlled-clearance piston gauge was characterized, and the uncertainty of the effective area of the gauge was evaluated in section 6 .

\section{Controlled-clearance gauge and the Heydemann-Welch model}

The pressure generated by a deadweight piston gauge at the reference level, $P$, is represented by the following equation:

$$
P=\frac{W}{A}+\left(\rho_{f}-\rho_{a}\right) \cdot g \cdot h
$$

where, $A$ is the effective area of the piston-cylinder assembly as a function of pressure and temperature; $\left(\rho_{f}-\right.$ $\left.\rho_{a}\right) \cdot g \cdot h$, is the head correction, with $\rho_{f}$ the density of the working fluid, $\rho_{a}$ the air density, $g$ the local acceleration due to gravity, and $h$ the vertical distance between the bottom of the piston and the reference level; and $W$ is the applied load on the piston. It is expressed as follows:

$$
W=M \cdot g \cdot\left(1-\frac{\rho_{a}}{\rho_{m}}\right)+\gamma \cdot C
$$

where, $M$ is the total mass of the loaded weights including the piston, $\rho_{m}$ is the mass density of the piston and weights, $\gamma$ is the surface tension of the working fluid and $C$ is the circumference of the piston where it emerges from the pressurizing fluid.

The equation of the effective area and its evaluation method depends on the type of piston gauge used as the standard. Fig. 1 shows the simplified schematic drawing of the piston gauges of (a) a simple type, and (b) a 


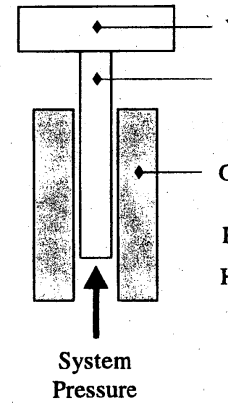

(a) simple type

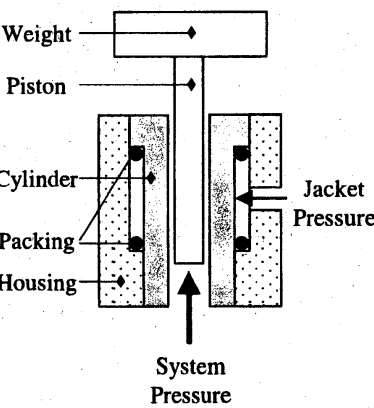

(b) Controlled-clearance type
Fig. 1 Schematic drawings of two different type piston gauges

controlled-clearance type, respectively. For a simple type piston gauge, the system pressure is applied to float the piston loaded by the weights. The effective area of the assembly for this type gauge is represented by the following equation, which assumes a linear pressure dependence:

$$
A(P, t)=A\left(0, t_{r}\right) \cdot(1+b \cdot P) \cdot\left\{1+\alpha_{s} \cdot\left(t-t_{r}\right)\right\}
$$

where, $A(P, t)$ is the effective area of the piston-cylinder assembly at pressure $P$ and temperature $t, A\left(0, t_{r}\right)$ is the effective area of the assembly at $0 \mathrm{~Pa}$ and at reference temperature, $t_{r}, b$ is the pressure distortion coefficient of the effective area, and $\alpha_{s}$ is the sum of $\alpha_{p}$ and $\alpha_{c}$, which are the linear thermal expansion coefficients for the piston and the cylinder respectively ${ }^{3)}$. Since the structure of this type gauge is simple, the operation is easy and the good reproducibility of pressure generated is the advantage. However, the clearance between the piston and cylinder of the gauge becomes larger generally depending on the pressure applied. Therefore, in high-pressure generation, the floated piston would be unstable because of the leakage from the clearance. For a controlled-clearance type gauge, another pressure referred to as a jacket pressure, $P_{j}$, is applied on the outer surface of the cylinder to adjust the clearance between the piston and cylinder. Therefore, the gauge can generate higher pressure compared with the simple type gauge. In the HeydemannWelch model, the effective area of the controlled-clearance type gauge is represented by

$$
\begin{array}{r}
A_{e}\left(P, P_{j}, t\right)=A_{p}\left(0,0, t_{r}\right) \cdot\left(1+b_{p} \cdot P\right) \\
\quad\left\{1+\alpha_{s} \cdot\left(t-t_{r}\right)\right\} \cdot\left\{1+d \cdot\left(P_{z}-P_{j}\right)\right\}
\end{array}
$$

where, $A_{e}\left(P, P_{j}, t\right)$ is the effective area of the pistoncylinder assembly at system pressure $P$, jacket pressure $P_{j}$ and temperature $t ; A_{p}\left(0,0, t_{r}\right)$ is the area of the piston determined from dimensional measurements adjusted to a reference temperature, $t_{r}$, at $P=0 \mathrm{~Pa}, P_{j}=0 \mathrm{~Pa}$; and $b_{p}$ is the pressure distortion coefficient of the piston

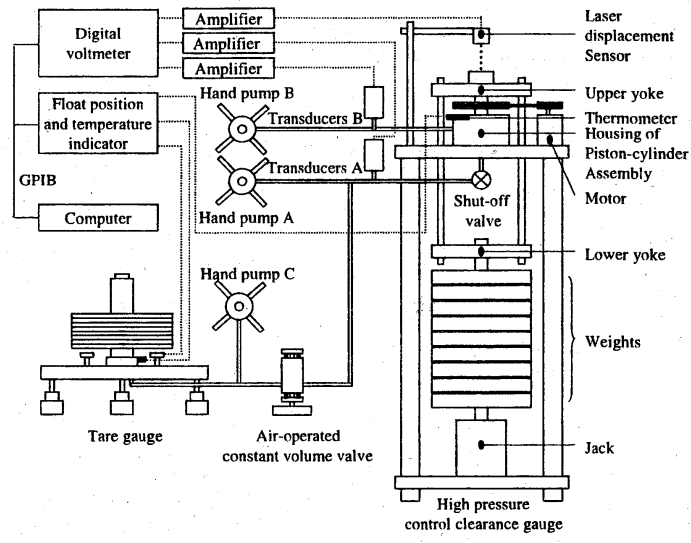

Fig. 2 Experimental apparatus

area determined from elasticity theory. $d$ is the jacket pressure coefficient, which is represented by the equation, $d=D+E \cdot W+F \cdot W^{2}$, where $D, E$, and $F$ are constants, and $W$ is the load. $P_{z}$ is the zero clearance jacket pressure, and is expressed by the equation, $P_{z}=P_{z 0}+S \cdot W+Q \cdot W^{2}$, where $P_{z 0}, S$, and $Q$ are constants. The other parameters in the equation are the same as for a simple gauge described above.

\section{Apparatus}

Fig. 2 shows a schematic drawing of the experimental apparatus and measuring devices used in this characterization. In the figure, a high-pressure controlled-clearance deadweight piston gauge is shown as the apparatus on the right side. The maximum pressure generated by the piston gauge is about $280 \mathrm{MPa}$. A piston-cylinder assembly is included and set into a special housing, which is placed on the top plate of the apparatus. The nominal effective area of the piston-cylinder assembly is $1.4 \times 10^{-5} \mathrm{~m}^{2}$. A motor rotates the piston in the cylinder clockwise to avoid the contact between them. The weights are shown in the middle part of the apparatus in the figure. The total mass of the weights is about $420 \mathrm{~kg}$. The load by the weights is applied on the piston through the lower and upper yokes. The number of applied weights can be adjusted by a jack.

The pressure to float the piston is generated by hand pump A shown in the figure. A shut-off valve near the inlet, underneath the housing, is used to isolate the piston gauge from the large volume of the pressurizing system during the fall-rate measurements. The jacket pressure used to control the clearance between the piston and cylinder is generated by hand pump B in the figure.

The piston gauge shown on the left side of the figure is referred to as a tare gauge used to measure the jacket pressure coefficient. In this characterization, a simple 
type piston gauge was used as the tare gauge. For the tare gauges, a piston-cylinder assembly of nominal effective area of $4.9 \times 10^{-6} \mathrm{~m}^{2}$ was used for the pressure range up to $200 \mathrm{MPa}$, and a piston-cylinder assembly of nominal effective area of $2.0 \times 10^{-6} \mathrm{~m}^{2}$ above $200 \mathrm{MPa}$. The pressure to float the piston of the tare gauge is generated by hand pump $\mathrm{C}$ in the figure. An air-operated constant volume valve is used to connect or disconnect the controlled-clearance piston gauge and the tare piston gauge $^{4)}$.

A laser displacement sensor was used to measure the floating position of the piston. The system pressure and the jacket pressure of the controlled-clearance gauge were measured with pressure transducers $\mathrm{A}$ and $\mathrm{B}$ as shown in the figure. The output signals of these sensors were amplified and detected by a digital voltmeter. The temperatures of the piston-cylinder assemblies of the controlledclearance piston gauge and the tare piston gauge were measured using two platinum resistance thermometers. The floating position of the tare gauge was measured using non-contact position sensors. These signals obtained from temperatures and the floating position of the tare gauge were processed by a float position and temperature indicator. A customized software program was written and used on a computer to collect all the data simultaneously through a GPIB bus and record on the hard-disk of the computer.

In this characterization, the reference temperature, $t_{r}$, was 23 degrees Celsius, and Spinnestic No.22 was used as the working fluid medium.

\section{Fall-rate measurements and Zero clearance jacket pressure}

In this section, the procedure and results of the fallrate measurements are described. The data obtained from the fall-rate measurements are analyzed using the Heydemann-Welch model to obtain the zero clearance jacket pressure, $P_{z}(W)$, with the uncertainty as a function of load, $W$.

For the controlled-clearance gauge, the fall-rate of the piston was measured for various load values on the piston, $W$, and various jacket pressure values, $P_{j}$. The characterization covers the pressure range from $37.5 \mathrm{MPa}$ to 288 $\mathrm{MPa}$, at jacket pressures up to $76 \mathrm{MPa}$. The measurement procedure is as follows. First, the weights are loaded on the piston, which generate the load, $W$. The system pressure, $P$, is increased to float the piston. After floating of the piston, the piston is rotated clockwise using the drive motor. Then, the jacket pressure, $P_{j}$, is adjusted
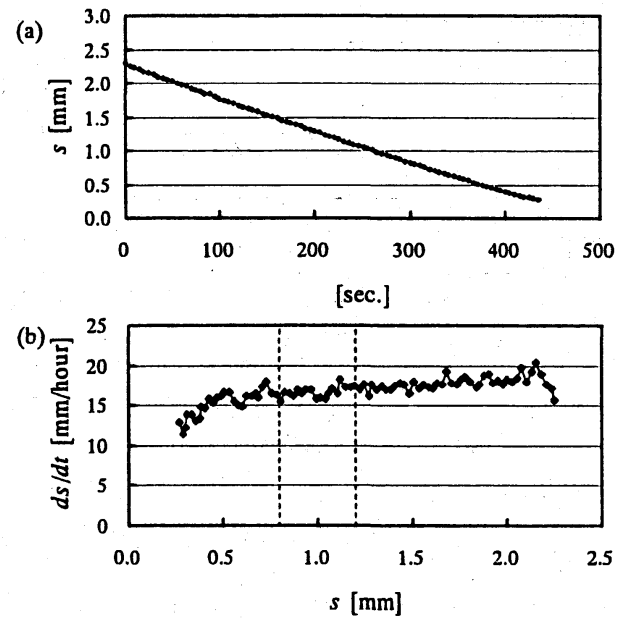

Fig. 3 Example of fall rate measurement, (a) floating piston position vs. time, (b) fall rate vs. the floating piston position, $W=4090 \mathrm{~N}$ and $P_{j}=0 \mathrm{MPa}$.

to the target value. The position of the piston is kept in the floating range to stabilize the pressure generated for almost 15 minutes. After the waiting time, the piston position is adjusted to the appropriate position using the hand pump, and the valve beneath the piston is closed to isolate the piston-cylinder assembly from the large volume of the connecting pressure lines and the hand pump. The piston position, the temperature of the piston-cylinder assembly, the system pressure and the jacket pressure are measured simultaneously every five seconds.

Fig. 3 shows a typical example of the fall-rate measurement. These data were collected at $W=4090 \mathrm{~N}$ and $P_{j}=0 \mathrm{MPa}$. Fig. 3 (a) shows the relation of the floating positions of the piston, $s$, which is sampled every 5 seconds, versus time. As shown in the figure, the floating piston position decreased from $2.3 \mathrm{~mm}$ to $0.3 \mathrm{~mm}$ over a time during of about 7 minutes. Fig. 3 (b) shows the relation of the fall-rates, $(d s / d t)$, which are calculated by taking the ratio of the difference in two successive piston positions to interval time, versus the floating piston position. All fall-rates for each $W$ and $P_{j}$ were measured between the piston positions of $2.0 \mathrm{~mm}$ and $0.3 \mathrm{~mm}$, and the data in the range between $1.2 \mathrm{~mm}$ and $0.8 \mathrm{~mm}$ were used to calculate the average fall-rate value from $(d s / d t)$. The position range is indicated by the dotted lines in the Fig. 3 (b). From this example, the average fall-rate, $(d s / d t)_{\text {ave }}$, is $16.55 \mathrm{~mm} /$ hour. Since this value occurs at the actual temperature of the measurement, $t$, it needs to be corrected to the value at the reference temperature, $t_{r},(d s / d t)_{\text {ave }}\left(t_{r}\right)$, using the temperature dependence of the viscosity of the working fluid as follows: $(d s / d t)_{\text {ave }}\left(t_{r}\right)=(d s / d t)_{\text {ave }}(t) \times \eta(t) / \eta\left(t_{r}\right)$, where 


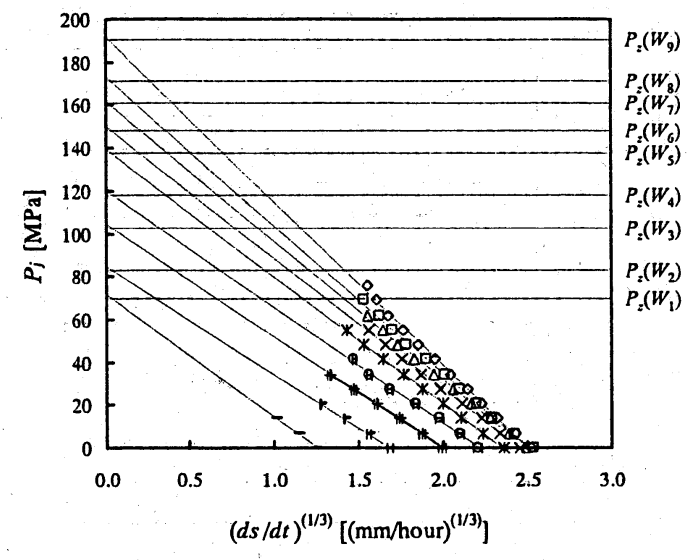

Fig. 4 Cube root of fall rate versus jacket pressure

$\eta(t)$ is the viscosity of the working fluid as a function of temperature $t$.

The fall-rate measurements were repeated over ranges of $W$ and $P_{j}$. In this characterization, $W$ was changed from $533.5 \mathrm{~N}$ to $4090 \mathrm{~N}$ in steps of $444.5 \mathrm{~N}$, and $P_{j}$ was changed from $0 \mathrm{MPa}$ to $76 \mathrm{MPa}$ in steps of $6.9 \mathrm{MPa}$. The cube root of the fall-rates as a function of $P_{j}$ for the nine loads is shown in Fig. 4. In the figure, there are nine different symbols that correspond to the value of the load, $W_{\mathrm{i}}$, which is approximately represented by the equation, $W_{\mathrm{i}}=(89+444.5 \times \mathrm{i})[\mathrm{N}]$, where i varied from 1 to 9 .

In order to determine the zero clearance jacket pressure for each $W$, the least-squares-best-fitting straight line is applied to the measured points obtained for the $W$, and extrapolated to the zero fall-rate as shown in the figure. The zero fall-rate intercept of the straight line is the zero clearance jacket pressure, $P_{z}\left(W_{\mathrm{i}}\right)$, measured for the $W_{\mathrm{i}}$. The relative standard deviation of each $P_{z}\left(W_{\mathrm{i}}\right)$ obtained from this method is generally less than $1 \times 10^{-2}$.

The set of measurements described above were repeated five times. Fig. 5 shows the mean value of the zero clearance jacket pressures measured, $P_{z}\left(W_{\mathrm{i}}\right)$, from five measurement sets. The relative standard deviations of the

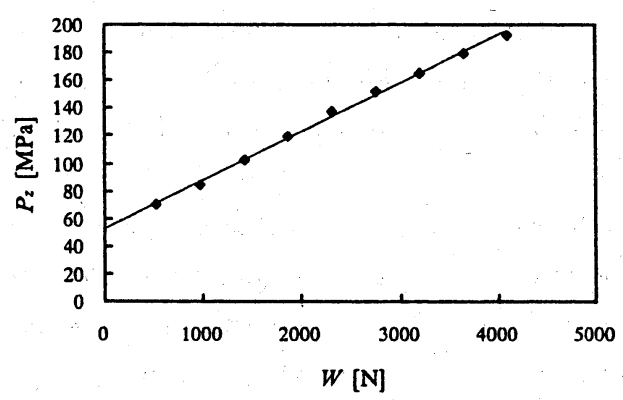

Fig. 5 Zero clearance jacket pressures, $P_{z}(W)$
Table 1 Evaluation result of coefficients of zero clearance jacket pressure function, $P_{z}=P_{z 0}+S \cdot W+Q \cdot W^{2}$, with standard uncertainties $(k=1)$

\begin{tabular}{|c|c|c|c|}
\hline$x_{i}$ & Value & $u\left(x_{\mathrm{i}}\right)(k=1)$ & Unit \\
\hline \hline$P_{z 0}$ & $5.25 \times 10^{7}$ & $1.2 \times 10^{6}$ & $\mathrm{~Pa}$ \\
\hline$S$ & $3.505 \times 10^{4}$ & $4.7 \times 10^{2}$ & $\mathrm{~Pa} \cdot \mathrm{N}^{-1}$ \\
\hline$Q$ & 0 & 0 & $\mathrm{~Pa} \cdot \mathrm{N}^{-2}$ \\
\hline
\end{tabular}

data are less than $3 \times 10^{-2}$.

In the Heydemann-Welch model, the zero clearance jacket pressure is defined as a function of $W$, and represented as follows: $P_{z}(W)=P_{z 0}+S \cdot W+Q \cdot W^{2}$. As shown in Fig. 5, the characteristics of points plotted from the measurement appear to be linear. Therefore, the coefficient, $Q$, is set to 0 , and a linear function, $P_{z}=P_{z 0}+S \cdot W$, is adopted as a reasonable representation of the data. The linear function is fitted to the data obtained from the measurements using a regression statistics program, and both coefficients, $P_{z 0}$ and $S$, are determined with their standard uncertainties $(k=1)$. Table 1 lists the coefficients of the zero clearance jacket pressure function with the standard uncertainties $(k=1)$.

\section{Jacket pressure coefficient}

To complete the characterization of the controlledclearance gauge using the Heydemann-Welch model, the controlled-clearance gauge was cross-floated against a tare gauge to evaluate the jacket pressure coefficient. This section describes the determination of the jacket pressure coefficient, $d(W)$, with the uncertainty as a function of load, $W$. The jacket pressure coefficient, $d$, in the HeydemannWelch model is obtained from the following equation ${ }^{2)}$ :

$$
d=\frac{1}{P} \cdot \frac{\delta P}{\delta P_{j}}
$$

where $P$ is the system pressure, and $P_{j}$ is the jacket pressure of the controlled-clearance gauge. From the equilibrium pressure of the cross-float measurement between the controlled-clearance gauge and the tare gauge, $P$ is calculated as the pressure generated by the tare gauge

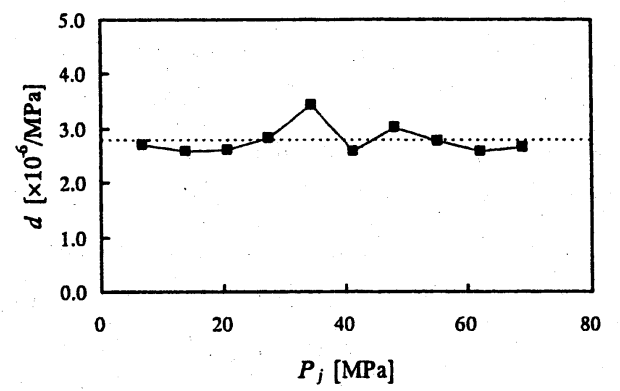

Fig. 6 Example of measurement result of jacket pressure coefficient, $d . W=3645.5 \mathrm{~N}$ 


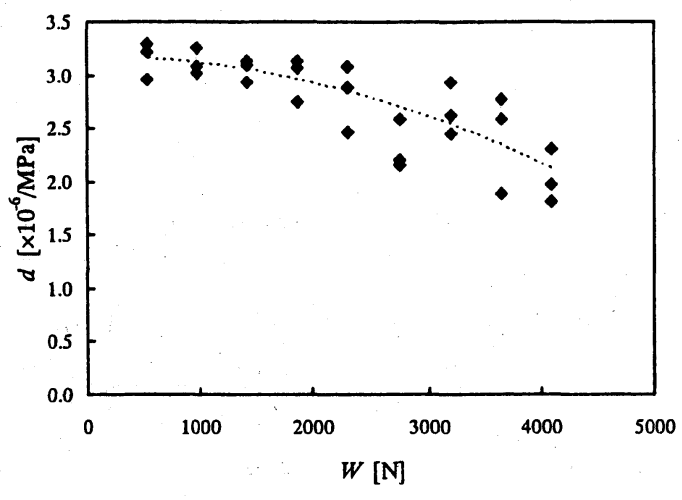

Fig. 7 Measured jacket pressure coefficient, $d(W)$

at the reference level using equations, (1), (2), and (3). $\delta P$ is obtained from the change in the system pressure by the change of $P_{j}$, using the cross float technique. For a given load on the controlled-clearance gauge, $W$, the jacket pressure coefficient, $d$, was measured as a function of $P_{j}$.

Fig. 6 shows an example of a measurement result of the jacket pressure coefficient, $d\left(P_{j}\right)$, where the load of $3645.5 \mathrm{~N}$ is applied on the piston. $P_{j}$ was changed from 0 $\mathrm{MPa}$ to $76 \mathrm{MPa}$ in steps of $6.9 \mathrm{MPa}$ approximately. The coefficient, $d$, was calculated using equation (5) from the difference in the system pressure between the closest two jacket pressure points, and plotted on the higher jacket pressure point of the range. The unit of the vertical axis is $\times 10^{-6} \mathrm{MPa}^{-1}$. As shown in the figure, the points plotted do not have the slope as a function of $P_{j}$. The average value of these points is calculated as a representative value. In the figure, the average value is shown by dotted line.

The $d$ measurements were performed over the same ranges of $W$ and $P_{j}$ as those used in the fall-rate measurement described in section 4 . For the tare gauge, the assemblies with nominal effective area of $4.9 \times 10^{-6} \mathrm{~m}^{2}$ and $2.0 \times 10^{-6} \mathrm{~m}^{2}$ were used below $200 \mathrm{MPa}$ and above 200 $\mathrm{MPa}$, respectively. A complete set of data was obtained for a total of nine loads used on the controlled-clearance gauge. The procedure was then repeated two more times. Fig. 7 shows the result of measurements of jacket pressure coefficient, $d\left(W_{\mathrm{i}}\right)$, as a function of $W_{\mathrm{i}}$, where $W_{\mathrm{i}}$ is explained in section 4 . All data from the three measurement sets are plotted.

In the Heydemann-Welch model, the jacket pressure coefficient is defined as a function of $W$, and represented as follows: $d(W)=D+E \cdot W+F \cdot W^{2}$. To determine the best fitting function for $d(W)$, for all of the available 27 points $(d, W)$, three functions were used. They are
Table 2 Evaluation result of coefficients of jacket pressure coefficient function, $d=D+E \cdot W+F \cdot W^{2}$, with standard uncertainties $(k=1)$

\begin{tabular}{|c||c|c|c|}
\hline$x_{i}$ & Value & $u\left(x_{\mathrm{i}}\right)(k=1)$ & Unit \\
\hline \hline$D$ & $3.155 \times 10^{-12}$ & $7.9 \times 10^{-14}$ & $\mathrm{~Pa}^{-1}$ \\
\hline$E$ & 0 & 0 & $\mathrm{~Pa}^{-1} \cdot \mathrm{N}^{-1}$ \\
\hline$F$ & $-6.43 \times 10^{-20}$ & $9.2 \times 10^{-21}$ & $\mathrm{~Pa}^{-1} \cdot \mathrm{N}^{-2}$ \\
\hline
\end{tabular}

(i) a linear function, $d=D+E \cdot W$, (ii) a quadratic function, $d=D+F \cdot W^{2}$, and (iii) a quadratic function, $d=D+E \cdot W+F \cdot W^{2}$. These three functions are fitted and examined using a regression statistics program. From the analytical evaluation, the quadratic function, (ii) $d=D+F \cdot W^{2}$, was selected as a reasonable representation of the data, and both coefficients, $D$ and $F$, were determined with the standard uncertainties $(k=1)$. The function is represented as a dotted line in Fig. 7. Table 2 lists the coefficients of the jacket pressure coefficient function with the standard uncertainties $(k=1)$.

\section{Characterization and Uncertainty evaluation}

The zero clearance jacket pressure, $P_{z}(W)$, and the jacket pressure coefficient, $d(W)$, are evaluated as a function of $W$ in the section 4 and 5 , respectively. In this section, $P_{z}(W)$ and $d(W)$ are converted to $P_{z}\left(P_{n}\right)$ and $d\left(P_{n}\right)$ using the following equation to examine the characteristics as a function of pressure:

$$
P_{n}=\frac{W}{A_{p}\left(0,0, t_{r}\right)}
$$

where $P_{n}$ is the nominal value of the pressure generated by the controlled-clearance piston gauge. In equation (4), the change in the effective area with the jacket pressure, $C_{j}$, is represented by the last term in equation (4):

$$
C_{j}=d \cdot\left(P_{z}-P_{j}\right)
$$

where $C_{j}$ is a function of $P_{n}$ and $P_{j}$, and is related to the crevice width between the piston and cylinder. It changes as a result of the change of the cylinder with the system and jacket pressures. In this paper, the next equation is used to represent the jacket pressure, $P_{j}$, as a function of $P_{n}$.

$$
P_{j}=\frac{A}{100} \cdot P_{n}+B
$$

where $A$ and $B$ are constants. $A$ is dimensionless and the unit of $B$ is $\mathrm{MPa}$. In measurements performed in this study, the maximum pressure of $P_{j}$ at each $P_{n}$ was determined approximately using equation (8) with $(A$, $B)=(25.0,10.0)$.

Fig. 8 shows the crevice term, $C_{j}$, calculated. The vertical axis shows the magnitude of the crevice term in parts 
Table 3 Values $(A, B)$ for function of jacket pressure, $P_{j}=(A / 100) \cdot P_{n}+B$

\begin{tabular}{|c||c|c|c|c|}
\hline & $(\mathrm{a})$ & (b) & (c) & (d) \\
\hline$A$ & 0.0 & 12.3 & 25.0 & 40.0 \\
\hline$B[\mathrm{MPa}]$ & 0.0 & 15.4 & 10.0 & 7.2 \\
\hline
\end{tabular}

per million, and the horizontal axis shows $P_{n}$. In the figure, four characteristic lines are plotted using four jacket pressure functions defined by equation (8) with the coefficients, $(A, B)$. Those coefficients, $(A, B)$, are $(A$, $B)=(\mathrm{a}):(0.0,0.0),(\mathrm{b}):(12.3,15.4),(\mathrm{c}):(25.0,10.0)$, and (d): $(40.0,7.2)$, as listed in Table 3. As shown in this figure, all representations of $C_{j}$ are quadratic functions of $P_{n}$, and the magnitude of $C_{j}$ is decreased when $P_{j}$ is increased at each $P_{n}$. In the figure, (a) shows the characteristic with $P_{j}=0$. For this characteristic, the $C_{j}$ at $P_{n}=0$ represents the relative difference between the areas of the piston and the effective area with zero system and jacket pressures, which is approximately $1.7 \times 10^{-4}$. Generally, the effective area is regarded as the average of the areas of piston and cylinder. Therefore, the relative difference between areas of the piston and the cylinder is estimated as about $3.4 \times 10^{-4}$, which corresponds the relative difference of about $1.7 \times 10^{-4}$ in radius of the circular effective area. Since the radius of the piston is about $2.1 \mathrm{~mm}$, the clearance between the piston and cylinder is estimated as about $0.36 \mu \mathrm{m}$ with zero system and jacket pressures. The coefficients, $(\mathrm{d}):(A, B)=(40.0,7.2)$, are calculated using the regression program, so that the variation of $C_{j}\left(P_{n}, P_{j}\right)$ with the jacket pressure determined by equation (8) using the coefficients and $C_{j}(0,0)$ with $P_{j}=\hat{0}$ are minimized in the pressure range from $37.5 \mathrm{MPa}$ to $288 \mathrm{MPa}$. The difference between the maximum and minimum values of the characteristic, $C_{j}$, with $(\mathrm{d})$ in the pressure range is about $2.0 \times 10^{-5}$

In equation (4), the term representing the change in area of the piston with the system pressure is

$$
C_{p}=b_{p} \cdot P_{n}
$$

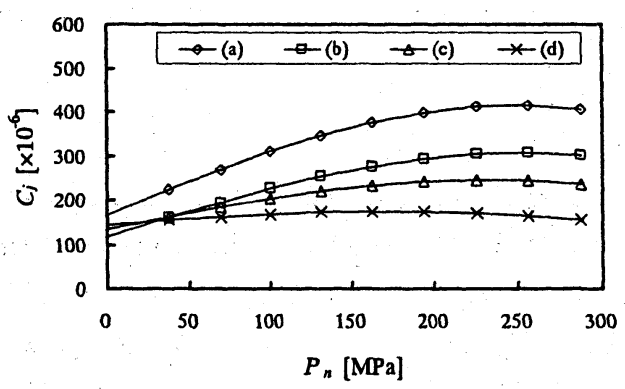

Fig. 8 Calculation result of correction term, $C_{j}=d \cdot\left(P_{z}-P_{j}\right)$
The total correction for the effective area with the system and jacket pressures, $C_{r}$, is calculated from

$$
C_{r}=C_{p}+C_{j}
$$

$C_{r}$, includes all the correction terms related to pressure in equation (4). Fig. 9 shows $C_{r}$ as a function of $P_{n}$ and $P_{j}$. In the figure, the characteristics of $C_{r}$ with four jacket pressure functions, which are the same as those described above, are plotted. The coefficients, $(\mathrm{b}):(A, B)=(12.3$, 15.4), is calculated using the regression program, so that the differences between $C_{r}\left(P_{n}, P_{j}\right)$ with the jacket pressure determined by equation (8) using the coefficients and $C_{r}(0,0)$ is minimized in the pressure range from $37.5 \mathrm{MPa}$ to $288 \mathrm{MPa}$. The variation between the maximum and minimum values of the characteristic, $C_{r}$, with (b) in the pressure range is about $4.5 \times 10^{-5}$.

In actual measurements, the coefficients of the jacket pressure, $P_{j}$, in equation $(8),(A, B)$, was changed between $(\mathrm{a}):(A, B)=(0.0,0.0)$ and $(\mathrm{c}):(A, B)=(25.0,10.0)$ for each $P_{n}$. In Fig. 9, the vertical range of $C_{r}$ between (a) and (c) shows the estimated change in the effective area of the controlled-clearance gauge for the system and jacket pressure ranges used in the actual measurements.

Table 4 shows the parameters and their uncertainties for the characterization of the effective area of the controlled-clearance gauge. The five components quantities considered are $A_{p}\left(0,0, t_{r}\right), b_{p}, \alpha_{s}, d$, and $P_{z}$. The uncertainties arising from $P, P_{j}$ and $\left(t-t_{r}\right)$ are sufficiently small, and are neglected here. $A_{p}\left(0,0, t_{r}\right)$ is measured dimensionally, and $b_{p}$ is calculated from elastic theory. The zero clearance jacket pressure, $P_{z}(W)$, and the jacket pressure coefficient, $d(W)$, are parameterized in the sections 4 and 5 in the usual way as described by Heydemann and Welch. The values of the coefficients of the functions are given in Table 1 and Table 2.

In Table $4, u\left(x_{\mathrm{i}}\right)$ are the uncertainties of the various measured quantities and $S\left(x_{i}\right) \equiv 1 / A_{e} \cdot\left(\partial A_{e} / \partial x_{i}\right)$ are

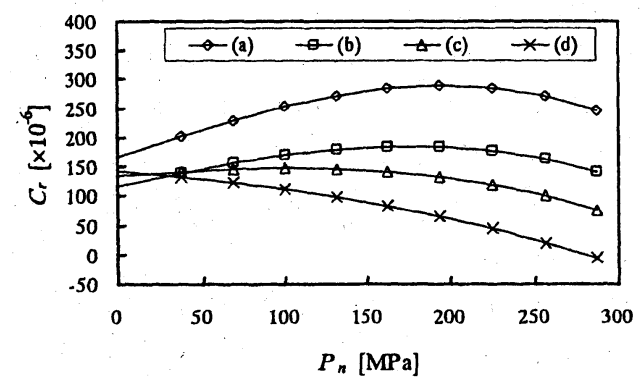

Fig. 9 Calculation result of correction term, $C_{r}=b_{p} \cdot P_{n}+d \cdot\left(P_{z}-P_{j}\right)$ 
Table 4 Parameters for characterization with standard uncertainties $(k=1)$ and sensitivity coefficients

\begin{tabular}{|c||c|c|}
\hline$x_{i}$ & $u\left(x_{i}\right)(k=1)$ & $S\left(x_{i}\right)$ \\
\hline \hline$A_{p}$ & $1.7 \times 10^{-10} \mathrm{~m}^{2}$ & $A_{p}^{-1} \cdot \mathrm{m}^{-2}$ \\
\hline$\alpha_{s}$ & $0.9 \times 10^{-6}{ }^{\circ} \mathrm{C}^{-1}$ & $\left(t-t_{r}\right)^{\circ} \mathrm{C}$ \\
\hline$b_{p}$ & $1.8 \times 10^{-14} \mathrm{~Pa}^{-1}$ & $P \mathrm{~Pa}$ \\
\hline$d$ & $u(D)+u(E) \cdot W+u(F) \cdot W^{2} \mathrm{~Pa}^{-1}$ & $\left(P_{z}-P_{j}\right) \mathrm{Pa}$ \\
\hline$P_{z}$ & $u\left(P_{z 0}\right)+u(S) \cdot W+u(Q) \cdot W^{2} \mathrm{~Pa}$ & $d \mathrm{~Pa}^{-1}$ \\
\hline
\end{tabular}

the sensitivity coefficients of those same quantities with respect to their contribution to the relative uncertainty of the effective area. The expanded $(k=2)$ relative uncertainty is obtained by the root sum of squares method ${ }^{5)}$ :

$$
\frac{U\left(A_{e}\right)}{A_{e}}=2 \cdot \sum_{i} \sqrt{\left\{u\left(x_{i}\right) \cdot S\left(x_{i}\right)\right\}^{2}}
$$

Fig. 10 represents the expanded $(k=2)$ relative uncertainty calculated by equation (11) for several system pressures, $P$, and jacket pressures, $P_{j}$, which are calculated by equation (8) using the coefficients listed in Table 3 . When (a): $(A, B)=(0,0)$ is used as the coefficients of jacket pressure, the maximum value of the relative uncertainty over the pressure range $37.5 \mathrm{MPa}$ to $288 \mathrm{MPa}$ occurs at $P=288$ $\mathrm{MPa}$, and is $96 \times 10^{-6}(k=2)$. In the actual measurements of this study, the maximum jacket pressure at each system pressure was determined by equation (8) with the coefficients $(\mathrm{c}):(A, B)=(25.0,10.0)$. When the coefficients are applied, then the expanded $(k=2)$ relative uncertainty in parts per million can be approximated by the following expression: $U\left(A_{e}\right) / A_{e}=26.5 \times 10^{-6}+3.22 \times 10^{-8} \cdot P-7.07 \times$ $10^{-11} \cdot P^{2}+1.31 \times 10^{-12} \cdot P^{3}(k=2)$, where $P$ is the system pressure in $\mathrm{MPa}$. The maximum value for this equation over the entire pressure range occurs at $P=288 \mathrm{MPa}$, and is $61 \times 10^{-6}(k=2)$. In this characterization, the coefficients $(\mathrm{d}):(A, B)=(40.0,7.2)$ were not applied actually. When the coefficients are applied, the expanded $(k=2)$ relative uncertainty are expected to decrease to $46 \times 10^{-6}$ at $P=288 \mathrm{MPa}$.

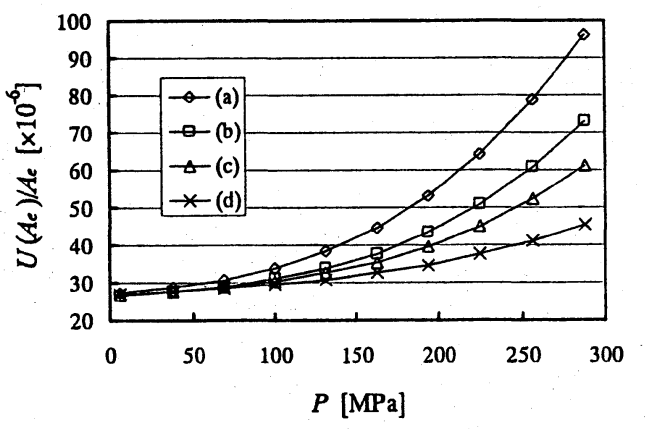

Fig. 10 Expanded $(k=2)$ relative uncertainties for the characterization of effective area of controlledclearance gauge
The uncertainty of $P, U(P) / P$, can be calculated from the uncertainties in equation (1). The uncertainty of the head correction $\left(\rho_{f}-\rho_{a}\right) \cdot g \cdot h$ can be reduced to a negligible amount by adjusting $h$ in the setup. Since the uncertainty of $W, U(W) / W$, is below $3 \times 10^{-6}$ in this experiment, and is negligible compared with $U\left(A_{e}\right) / A_{e}$ analyzed. Therefore, in this case, it can be regarded that $U(P) / P$ is almost identical with $U\left(A_{e}\right) / A_{e}$.

\section{Summary}

A study on the characterization of a controlledclearance piston gauge was performed. The characterization covers the pressure range from $37.5 \mathrm{MPa}$ to 288 $\mathrm{MPa}$, at the jacket pressures up to $76 \mathrm{MPa}$. In this study, the Heydemann-Welch model was used to characterize the controlled-clearance piston gauge as a primary pressure standard. The parameters needed for the model were evaluated from the measurements for the characterization. The results obtained are as follows: (1), The best jacket pressure function to keep the clearance between the piston and cylinder constant was determined from the measurement. (2) The dimensional clearance between the piston and cylinder was estimated. (3) The uncertainty of the effective area of the piston-cylinder used for the gauge was evaluated by the function of the system and jacket pressures.

The method used for the characterization will be examined for the improvement of the high-pressure standard at NMIJ/AIST in Japan as one of the evaluation methods. By the improvement, it is expected to reduce the uncertainty of the pressure standard provided for our industry.

\section{Acknowledgements}

This research was performed at NIST. One of the authors (Kobata) would like to thank the members of pressure and vacuum group at NIST who helped to carry out this research.

\section{References}

1) V. E. Bean: NIST Pressure Calibration Service, NIST special Publication 250-39, NIST (1994)

2) P. L. M. Heydemann and B. E. Welch: Part 3. Piston Gages - Experimental Thermodynamics of Non-reacting Fluids, Experimental Thermodynamics, II, 147/202, International Union of Pure and Applied Chemistry (1975)

3) NCSL RIPS-4: Deadweight Pressure Gauges (1998)

4) W. Markus: A Constant Volume Valve, The Review of Scientific Instruments, 43-1, 158/159 (1972)

5) B. N. Taylor and C. E. Kuyatt: Guidelines for Evaluating and Expressing the Uncertainty of NIST Measurement Results, NIST Technical Note 1297, 1994 Edition, (1994) 


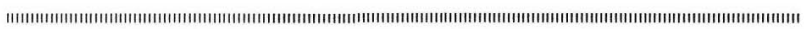

Tokihiko Ковата (Member)

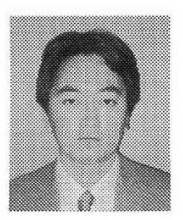

He received his M.E. and Dr. Eng. degrees in applied physics from the University of Tsukuba, Ibaraki, Japan, in 1992 and 1995, respectively. He has been engaged in research on standards and measurement of pressure at the National Metrology Institute of Japan, AIST.

\section{James W.SCHMIDT}

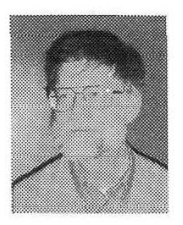

He received M.S. and $\mathrm{PhD}$ in physics both from the University of Delaware in 1978 and 1981, respectively. He has been engaged in research on the thermodynamic properties of fluids and on pressure standards at NIST (formerly NBS) since 1981.

\section{Douglas A.OLson}

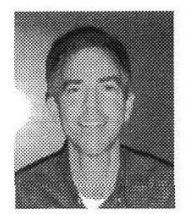

$\mathrm{He}$ received his $\mathrm{MS}$ and $\mathrm{PhD}$ degrecs in mechanical engineering from the Massachusetts Institute of Technology in 1979 and 1987, respectively. He has been engaged in research on standards and measurement of pressure at the National Institute of Standards and Technology, Gaithersburg, MD, USA.

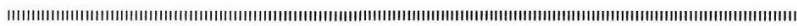

